\title{
RELATIONSHIP BETWEEN INDEX-TO-RING FINGER LENGTH RATIO (2D:4D) AND WOMAC OSTEOARTHRITIS INDEX IN WOMEN WITH KNEE OSTEOARTHRITIS
}

\author{
SHAFUZAIN AB RAHMAN ${ }^{1}$, NURUL FAKHRI AMIR ${ }^{1}$ \\ and MOHD ARIFF SHARIFUDIN ${ }^{2 *}$ \\ ${ }^{1}$ Department of Orthopaedics, School of Medical Sciences, Universiti Sains Malaysia, \\ Kubang Kerian, Kelantan, Malaysia \\ ${ }^{2}$ Faculty of Medicine, Universiti Sultan Zainal Abidin, Kuala Terengganu, \\ Terengganu, Malaysia \\ *E-mail: ariffsharifudin@unisza.edu.my
}

Accepted 4 November 2021, Published online 30 November 2021

\begin{abstract}
The difference in the index-to-ring finger length ratio (2D:4D) is associated with human behavior, physical capability, and risk of developing knee osteoarthritis in women. We aimed to determine the relationship between 2D:4D, Western Ontario and McMaster (WOMAC) osteoarthritis index, and body mass index (BMI) in women with mild to moderate knee osteoarthritis. A cross-sectional study was conducted on 118 women with Kellgren-Lawrence grade 2-3 knee osteoarthritis using the WOMAC questionnaire. The length of the index and ring fingers were measured using right-hand posterioanterior view plain radiographs. The participants were classified into three groups: type I (index finger longer than ring finger), type II (equal length), and type III (index finger shorter than ring finger). BMI was determined using standard measurements and calculations. There was no significant mean difference between the mean total WOMAC score and each of its components with the 2D:4D ratio. BMI correlated significantly with symptoms of knee osteoarthritis and the total WOMAC score $(P<0.001)$. There was a significant relationship between the $2 \mathrm{D}: 4 \mathrm{D}$ ratio and BMI $(P=0.015)$. No relationship was found between the 2D:4D ratio and WOMAC score. However, there was a positive correlation between the WOMAC score and BMI. The 2D:4D ratio had a significant relationship with BMI.
\end{abstract}

Key words: 2D:4D ratio, osteoarthritis knee, finger length, female gender, WOMAC score

\section{INTRODUCTION}

The prevalence among women with radiological evidence of knee osteoarthritis (OA) is higher compared to males. Physical disability is one of the main issues with osteoarthritis of the lower limb (Torres et al., 2006; Haugen et al., 2011). OA is one of the most common joint disorders that lead to physical disability in the elderly. Although elderly women experience a lower rate of chronic disease and longer life span than men, studies reported that they have a lower quality of life due to functional limitation and physical disability (McAlindon et al., 1993; Kobayashi et al., 2016; White et al., 2016). Disability among gender differences remains unexplained although some studies have tried to associate it with

* To whom correspondence should be addressed. demographic factors, chronic diseases, osteoarthritis, and other joint diseases.

The Western Ontario and McMaster Universities Arthritis Index (WOMAC) is widely used in the evaluation of knee and hip osteoarthritis. Since it was developed in 1982 at the Western Ontario and McMaster Universities, WOMAC has been extensively used in epidemiological and observational studies, and to assess improvement in patients with post-treatment intervention. Its use has been validated in many medium, including through questionnaires and via phone or computer. The WOMAC has been proven to be reliable, responsive, and efficient (McConnell et al., 2001). It is a selfadministered questionnaire consisting of 24 items divided into 3 subscales, including pain (5 subsets), stiffness ( 2 subsets), and disability (17 subsets). The scores are summed for items in each subscale on the Likert version: pain (0-20), stiffness (0-8), and 
disability (0-68). The higher the score indicates worsening in pain, stiffness, and limited functional ability. The WOMAC score has been translated and validated in Bahasa Malaysia (Ab-Rahman et al., 2020).

Anthropological studies have suggested that there is consistency between gender differences with the ratio of the lengths of the index finger (2D) and the ring finger (4D) (Manning et al., 1998; Manning et al., 2000; Manning and Taylor, 2001; Honekopp \& Watson, 2010). Men typically have 4D that is longer than their $2 \mathrm{D}$, compared to women who in general have an equal length or longer 2D. The discrepancy between the length of $2 \mathrm{D}$ and 4D reflects exposure to prenatal testosterone. A low 2D:4D ratio indicates higher exposure in utero testosterone (Manning et al., 1998; Lutchmaya et al., 2004; Mcintyre et al., 2006; Richards et al., 2017). The 2D:4D ratio can be classified into three types. Type I is when the $2 \mathrm{D}$ is longer than the $4 \mathrm{D}$, equal length is seen in type II, and the $2 \mathrm{D}$ is shorter than the $4 \mathrm{D}$ in type III (Zhang et al., 2008). Previous studies on the risk of knee OA and the 2D:4D ratio have shown inconsistent results, with some studies concluding that type III is associated with risk of knee OA in women (Zhang et al., 2008; Ferraro et al., 2010; Hussain et al., 2014; Kalichman et al., 2018).

The current study aimed to determine the relationship between symptomatic knee OA based on the components of the WOMAC score and the 2D:4D ratio, specifically in women. Functional disability in knee OA has been reported to be positively correlated with high BMI. Therefore, we also aimed to determine any correlation between the WOMAC score and BMI, and any significant correlation between $\mathrm{BMI}$ and the $2 \mathrm{D}: 4 \mathrm{D}$ ratio.

\section{MATERIALS AND METHODS}

The study was designed as a comparative crosssectional study involving female patients with primary knee OA. The participants were selected among patients aged 50 years and above with primary knee OA, Kellgren and Lawrence (K-L) grades 2 or 3 , presenting to the arthroplasty clinic of a tertiary institution. We only included grades 2 and 3 as they are categorized as a moderate type of OA. Patients who had received any form of intraarticular knee injection within three months before assessment were excluded from the study. Those with post-traumatic osteoarthritis or other types of arthritis including rheumatoid arthritis, gouty arthritis, psoriatic arthritis, and post-septic arthritis were also excluded. Other exclusion criteria were patients who had undergone knee replacement surgery, patients with hand deformity or history of digit fracture, or patients with a history of major psychiatric illness, dementia, or medical illness unfit for assessment.

The participants' weight was measured to the nearest $0.1 \mathrm{~kg}$ using a beam balance scale and their height was measured to the nearest $1 \mathrm{~mm}$ using a stadiometer. Both were measured with their shoes off. BMI was calculated as weight in kilograms divided by the square of height in meters. The whole cohort of participants had completed the WOMAC score. Every question has been fully understood by the participant and then marked. This was to avoid any bias caused by incomplete understanding or inability to read the written form. On average, it took 10 to 15 min to complete the WOMAC score. The participants were subjected to a right hand posteroanterior (PA) view plain radiograph. Radiation dose (effective dose) for plain radiograph of the extremities was as low as $0.001 \mathrm{mSV}$ (miliSievert). Hence, the risk of any malignancy was negligible. The participant was seated adjacent to the radiographic table. The hand radiograph was taken in a prone position with no angulation at the wrist. The central beam was directed at the third metacarpophalangeal joint in the expanded hand. Images were obtained using a small focal point and a detail cassette. The exposures were $48 \mathrm{kV}, 3.2 \mathrm{~mA} . \mathrm{s}$, and the distance was $90 \mathrm{~cm}$ from the source of the image. Each film was scanned and was saved into a PAC system. The right hand was used for the radiographic measurement of the 2D:4D ratio based on the previous study which reported that the $2 \mathrm{D}: 4 \mathrm{D}$ ratio is symmetrical between both sides and is not related to hand dominance (Manning et al., 2005; Robertson et al., 2008). The length of the 2D and 4D were measured from the midpoint of the base of the proximal phalanx to the midpoint of the tip of the distal phalanx to determine the $2 \mathrm{D}: 4 \mathrm{D}$ ratio (Figure 1). All data were analyzed using IBM Statistical Package for Social Science (SPSS) version 22.0.

\section{RESULTS AND DISCUSSION}

There were 118 participants recruited for the study. The mean (SD) age of the participants was 62.1 (6.79) years old, ranging between 50 to 80 years old. The majority of the participants were in the age groups between 50 to 59 years old, and 60 to 69 years old ( $n=52$ in each age group). The mean (SD) BMI was 28.9 (4.54), with the majority of participants being either obese $(42 \%)$ or overweight $(46 \%)$. Type III finger patterns contributed the highest percentage of participants involving nearly half of them $(n=56,47 \%)$. This was followed by type I $(29 \%)$ and type II (24\%). 


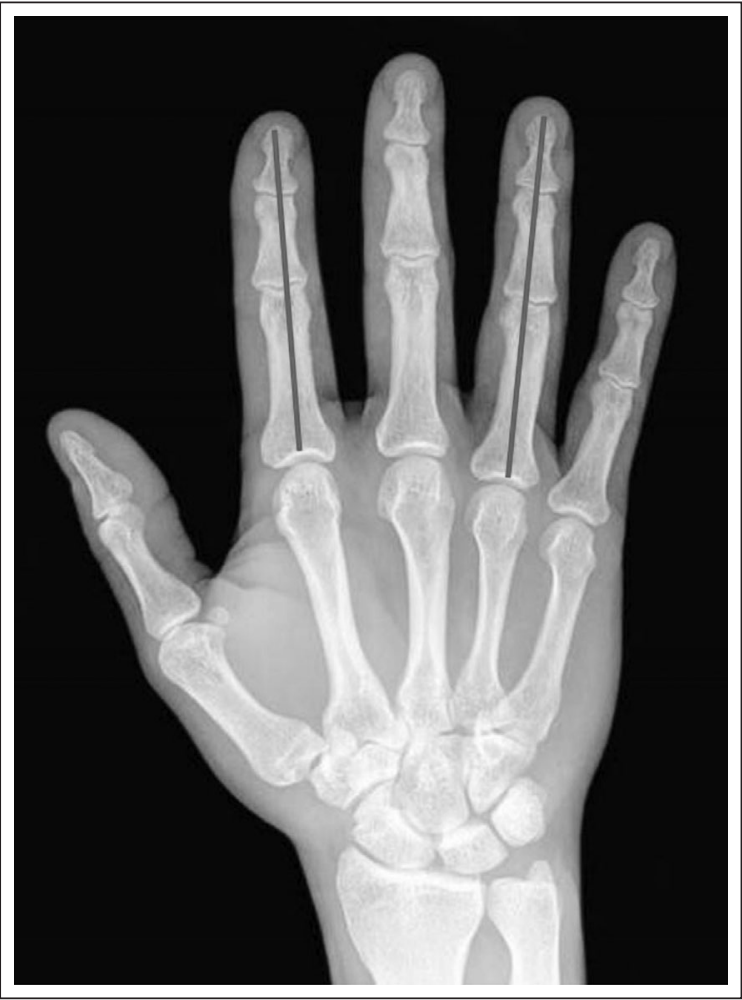

Fig. 1. Measuring the length of the index finger and the ring finger on a plain radiograph.

\section{The 2D:4D ratio and fetal androgen exposure}

Ferraro et al. (2010) and Zhang et al. (2008) reported a nearly similar distribution of the 2D:4D ratio in women with knee $\mathrm{OA}$ in their samples.
Community database studies on radiological assessment in classifying 2D:4D patterns in Caucasian adults showed the prevalence of a definite type III pattern on either hand was $37.29 \%$ in women, and $60.88 \%$ in men. Men were three times as likely to have a type III pattern than women (Honekopp et al., 2006; Robertson et al., 2008; Richards et al., 2017; Kalichman et al., 2018). The 2D:4D ratio is an indirect biological marker of pain in knee osteoarthritis that highlights dimorphic anatomical gender differences in males and females. Fetal androgen exposure is of interest to researchers because it has been playing a role in neurological development and was thought to have vital contributions to biological and psychological gender differentiation.

\section{Relationship between the $2 D: 4 D$ ratio and symptomatic knee OA}

One-way ANOVA was used to compare the mean of total WOMAC score and its components between the types of $2 \mathrm{D}: 4 \mathrm{D}$ ratio patterns. There was no significant difference between the three patterns for the total WOMAC score and all its components (Table 1). There was no previous study that explained the relationship between the $2 \mathrm{D}: 4 \mathrm{D}$ ratio and disability following knee osteoarthritis among women. On the other hand, there were reports that the type III pattern was found to be positively associated with athletic and spatial ability. It has been associated with achievement in many physically demanding and competitive sports. The researchers also indicated that individuals with type III patterns

Table 1. Comparison of the mean total WOMAC score and its components between the types of $2 \mathrm{D}: 4 \mathrm{D}$ pattern

\begin{tabular}{|c|c|c|c|c|}
\hline Type & $n$ & Mean (SD) & F-stat (df) & $P$-value \\
\hline \multicolumn{5}{|c|}{ a. The mean of total WOMAC score between the types of $2 D: 4 D$ ratio pattern } \\
\hline Type I & 34 & $34.9(11.19)$ & $0.97(2)$ & 0.383 \\
\hline Type II & 28 & $38.7(11.42)$ & & \\
\hline Type III & 56 & $36.2(9.92)$ & & \\
\hline \multicolumn{5}{|c|}{ b. The mean of score for pain component between the types of $2 D: 4 D$ ratio pattern } \\
\hline Type I & 34 & $6.6(3.86)$ & $1.03(2)$ & 0.360 \\
\hline Type II & 28 & $8.0(3.83)$ & & \\
\hline Type III & 56 & $7.5(3.87)$ & & \\
\hline \multicolumn{5}{|c|}{ c. The mean of score for joint stiffness component between the types of $2 D: 4 D$ ratio pattern } \\
\hline Type I & 34 & $2.3(1.47)$ & $0.58(2)$ & 0.560 \\
\hline Type II & 28 & $2.7(1.28)$ & & \\
\hline Type III & 56 & $2.5(1.41)$ & & \\
\hline \multicolumn{5}{|c|}{ d. The mean of the score for disability component between the types of $2 D: 4 D$ ratio pattern } \\
\hline Type I & 34 & $26.0(7.46)$ & $0.77(2)$ & 0.464 \\
\hline Type II & 28 & $28.0(7.75)$ & & \\
\hline Type III & 56 & $26.2(6.35)$ & & \\
\hline
\end{tabular}

A one-way ANOVA test was applied. 
were more likely to take risks, more active, and have a less functional disability in daily activities in the younger population. This was believed to lead to knee trauma and eventually OA (Manning et al., 2001; Hampson et al., 2008; Haugen et al., 2011; Hussain et al., 2014; Kalichman et al., 2018). Although the relationships between knee trauma and the development of post-traumatic OA remain poorly understood, it is clear that joint dislocation, articular injury, ligament, and meniscus tears in an active person may increase the risk of knee OA, which can cause functional disability later in life (McAlindon et al., 1993; White et al., 2006; Haugen et al., 2011; Hussain et al., 2014; Kobayashi et al., 2016; Torres et al., 2016; Ab-Rahman et al., 2020). Based on previous studies, we presumed that the type III pattern in women is more prone to functional disability at a more advanced age compared to other groups. However, results from the WOMAC score in the physical disability component in the current study did not support our hypothesis.

Inconsistent results on the relationship between the feeling of pain and the 2D:4D ratio had been reported in the past. Keogh et al. (2007) reported that women with type I pattern have a lower pain threshold than men with similar finger patterns. This was not supported by Yamamotova et al. (2009), where the rate of pain threshold was not significant in women. They are more sensitive to experimentallyinduced pain and their responses to noxious stimuli in comparison with men (Fillingim \& Ness, 2000). Although there were limited studies on the relationship between pain response to the $2 \mathrm{D}: 4 \mathrm{D}$ ratio, we presumed that type I pattern has more sensitivity to pain with a higher WOMAC score in the pain component. It would be related to a lower prenatal testosterone exposure during birth and activation effect from circulating hormone during adulthood associated with low pain threshold (Fillingim \& Ness, 2000). However, the current study did not find results to support a significant correlation between female finger traits and higher pain sensitivity. Acute pain is different from chronic pain, which arises from psychological states, serves no biological purpose, and has no recognizable end-point. Other studies also showed that the prevalence of chronic pain was more significant with advanced age, geographical and cultural variation (Elliot et al., 2002; Breivik et al., 2006).

Although type III finger pattern was associated with a higher risk for knee OA in women (Zhang et al., 2008; Ferraro et al., 2009), our results showed there was no correlation with functional disability. The difference in finger patterns does not have an influence on functional disability and pain in women with knee OA. This finding corresponds to the previous study by Haugen et al. (2011) that reported no significant correlation between the severity of symptoms in knee OA and the 2D:4D pattern.

\section{Correlation between BMI and the WOMAC score}

A significant correlation between the total WOMAC score and BMI among participants in the current study was found when Pearson's Correlation Coefficient was applied (Figure $2 \&$ Table 2). A positive linear relationship (moderate correlation) between total WOMAC and BMI was observed $(P<0.001, r=0.643)$. However, BMI was confirmed as

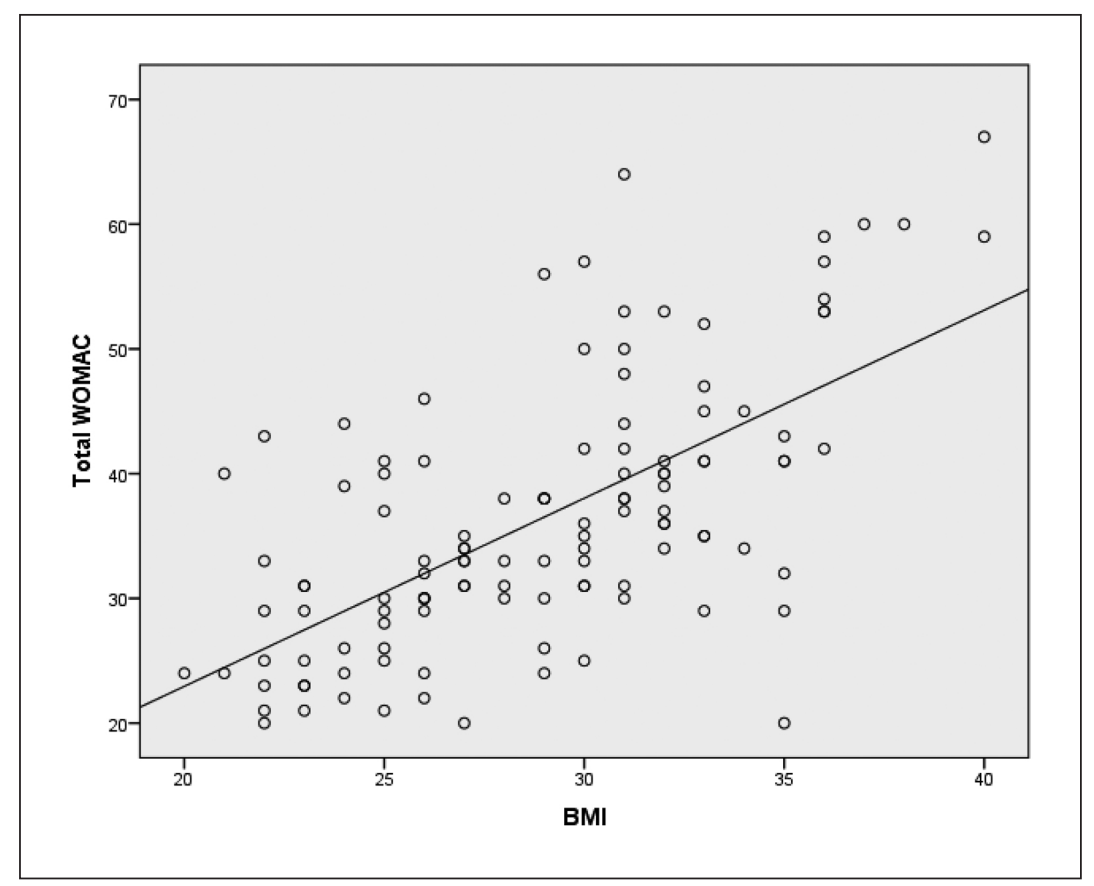

Fig. 2. Correlation between total WOMAC score and BMI $(r=0.643, P<0.001)$. 
Table 2. Descriptive statistics and correlation coefficients for study variables

\begin{tabular}{lccccc}
\hline Variable & Mean & SD & 1 & 2 & 3 \\
\hline 1. BMI & 28.9 & 4.5 & & & \\
2. WOMAC & 36.4 & 10.7 & $0.643^{* *}$ & & \\
3. 2D4D Ratio & 1.0 & 0.1 & -0.044 & -0.052 & \\
\hline
\end{tabular}

** $p<0.01$ (2-tailed); $n=118 ; \mathrm{SD}=$ standard deviation.

Table 3: Comparison of mean BMI between the types of 2D:4D patterns

\begin{tabular}{lllcr}
\hline Type & $n$ & Mean (SD) & F-stat (df) & $P$-value \\
\hline Type I & 34 & $27.1(3.92)$ & $4.38(2)$ & 0.015 \\
Type II & 28 & $30.2(4.31)$ & & \\
Type III & 56 & $29.4(4.74)$ & & \\
\hline
\end{tabular}

A one-way ANOVA test was applied.

not a confounding factor in the relationship between WOMAC and 2D:4D which was tested in ANCOVA analysis. Further analysis found a significant association between the types of 2D:4D pattern and BMI (Table 3). The majority of participants with the highest mean BMI had a type II 2D:4D pattern.

Similar findings were reported in previous studies (Felson et al., 1992; Coggon et al., 2001). A higher body weight leads to an increment in mechanical stress on the joints. BMI is one of the modifiable risk factors that can reduce progression and physical disability in knee OA. Therefore, a proper weight reduction program should be recommended for elderly women with knee OA. It was suggested that a two-point BMI reduction or a $5 \mathrm{~kg}$ loss could effectively reduce problems related to knee OA (Felson et al., 1997; Coggon et al., 2001; Elbaz et al., 2011).

Even though there was a high correlation between WOMAC and BMI $(P<0.001, r=0.643)$, BMI was confirmed as not a confounding factor in the relationship between WOMAC and the 2D:4D ratio. There was also a significant association between a higher BMI and the different 2D:4D ratios. Ironically, the type II finger pattern, which has the least number of participants $(n=28,24 \%)$, has more association with higher BMI. We believe that poor functional ability, pain, and less physical activity among women with knee OA lead to a higher BMI. There is also a possibility that female finger pattern is related to eating behavior with increased food intake during adolescence and puberty (Klump et al., 2006). In contrast, an eating disorder study by Quinton et al. (2011) showed that the type III pattern has a strong association with anorexia in females.

\section{CONCLUSION}

Based on our results, we suggest that weight reduction should be more emphasized for a targeted group of women with type II finger patterns. Early intervention may reduce and delay the physical disability due to the progression of the disease later. However, with a small group of participants in the type II pattern in the current study, the outcome may not have a significant impact. It is hoped that further research will contribute to improving our understanding of the mechanism responsible for this association.

\section{ACKNOWLEDGEMENTS}

The current study was part of a dissertation by one of the authors submitted in partial fulfillment of the requirements for the degree of Master of Medicine (Orthopaedics), and part of ongoing research works involving all the authors.

\section{REFERENCES}

Ab-Rahman, S., Narhari, P., Sharifudin, M.A. \& Shokri, A.A. 2020. Western Ontario and McMaster Universities (WOMAC) Osteoarthritis Index as an assessment tool to indicate total knee arthroplasty in patients with primary knee osteoarthritis. IIUM Medical Journal Malaysia, 19(3): 47-53. 
Breivik, H., Collett, B., Ventafridda, V., Cohen, R. \& Gallacher, D. 2006. Survey of chronic pain in Europe: prevalence, impact on daily life, and treatment. European Journal of Pain, 10(4): 287 333.

Coggon, D., Reading, I., Croft, P., Mclaren, M., Barrett, D. \& Cooper, C. 2001. Knee osteoarthritis and obesity. International Journal of Obesity and Related Metabolic Disorders, 25(5): 622627.

Elbaz, A., Debbi, E.M., Segal, G., Haim, A., Halperin, N., Agar, G., Nor, A. \& Debi, R. 2011. Sex and body mass index correlate with Western Ontario and McMaster Universities Osteoarthritis Index and quality of life scores in knee osteoarthritis. Archives of Physical Medicine and Rehabilitation, 92(10): 1618-1623.

Elliott, A., Smith, B. \& Hannaford, P. 2002. The course of chronic pain in the community: results of a 4-year follow-up study. Pain, 99(1-2): 299-307.

Felson, D.T., Zhang, Y., Hannan, M.T., Naimark, A., Weissman, B., Aliabadi, P. \& Levy, D. 1997. Risk factors for incident radiographic knee osteoarthritis in the elderly: The Framingham Study. Arthritis Rheumatism, 40(4): 728-733.

Ferraro, B., Wilder, F.V. \& Leaverton, P.E. 2010. Site specific osteoarthritis and the index to ring finger length ratio. Osteoarthritis Cartilage, 18(3): 354-357.

Fillingim, R.B. \& Ness, T.J. 2000. Sex-related hormonal influences on pain and anaelgesics responses. Neuroscience and Biobehavioral Reviews, 24(4): 485-501.

Fink, B., Neave, N. \& Manning, J.T. 2003. Second to fourth digit ratio, body mass index, waist-to-hip ratio, and waist-to-chest ratio: their relationship in heterosexual men and women. Annals of Human Biology, 30(6): 728-738.

Hampson, E., Ellis, C.L. \& Tenk, C.M. 2008. On the relation between 2D:4D and sex-dimorphic personality traits. Archives of Sexual Behavior, 37(1): 133-144.

Haugen, I.K., Niu, J., Aliabadi, P., Felson, D. \& Englund, M. 2011. The associations between finger length pattern, osteoarthritis, and knee injury: data from the Framingham community cohort. Arthritis \& Rheumatology, 63(8): 22842288.

Hönekopp, J. \& Watson, S. 2010. Meta analysis of digit ratio 2D:4D shows greater sex difference in the right hand. American Journal of Human Biology, 22(5): 619-630.

Honekopp, J., Manning, J.T. \& Muller, C. 2006. Digit ratio (2D:4D) and physical fitness in males and females: evidence for effects of prenatal androgens on sexually selected traits. Hormones and Behaviour, 49(4): 545-549.
Hussain, S.M., Wang, Y., Muller, D.C., Wluka, A.E., Giles, G.G., Manning, J.T., Graves, S. \& Cicuttini, F.M. 2014. Association between index-to-ring finger length ratio and risk of severe knee and hip osteoarthritis requiring total joint replacement. Rheumatology, 53(7): 1200-1207.

Kalichman, L., Batsevich, V. \& Kobyliansky, E. 2018. 2D:4D finger length ratio and radiographic hand osteoarthritis. Rheumatology International, 38(5): 865-870.

Keogh, E., Mounce, C. \& Brosnan, M. 2007. Can a sexually dimorphic index of prenatal hormonal exposure be used to examine cold pressor pain perception in men and women? European Journal of Pain, 11(2): 231-236.

Klump, K.L., Gobrogge, K.L., Perkins, P.S., Thorne, D., Sisk, C.L. \& Breedlove, S.M. 2006. Preliminary evidence that gonadal hormones organize and activate disordered eating. Psychological Medicine, 36(4): 539-546.

Kobayashi, S., Pappas, E., Fransen, M., Refshauge, K. \& Simic, M. 2016. The prevalence of patellofemoral osteoarthritis: a systematic review and meta-analysis. Osteoarthritis and Cartilage, 24(10): 1607-1707.

Lutchmaya, S., Baron-Cohen, S., Raggatt, P., Knickmeyer, R. \& Manning J.T. 2004. 2nd to 4th digit ratios, fetal testosterone and estradiol. Early Human Development, 77(1-2): 23-28.

Manning, J.T., Scutt, D., Wilson, J. \& Lewis-Jones, D.I. 1998. The ratio of 2 nd to 4 th digit length: a predictor of sperm numbers and concentrations of testosterone, luteinizing hormone and oestrogen. Human Reproduction, 13(11): 30003004.

Manning, J.T, Barley, L., Walton, J., Lewis-Jones, D.I., Trivers, R.L., Singh, D., Thornhill, R., Rohde, P., Bereczkei, T., Henzi, P., Soler, M. \& Szwed, A. 2000. The 2nd:4th digit ratio, sexual dimorphism, population differences, and reproductive success. Evidence for sexually antagonistic genes? Evolution and Human Behavior, 21(3): 163-183.

Manning, J.T. \& Taylor, R.P. 2001. Second to fourth digit ratio and male ability in sport: implications for sexual selection in humans. Evolution and Human Behavior, 22(1): 61-69.

Manning, J.T., Fink, B., Neave, N. \& Caswell, N. 2005. Photocopies yield lower digit ratios (2D:4D) than direct finger measurements. Archives of Sexual Behaviour, 34(3): 329-333.

McAlindon, T.E., Cooper, C., Kirwan, J.R. \& Dieppe, P.A. 1993. Determinants of disability in osteoarthritis of the knee. Annals of the Rheumatic Diseases, 52(4): 258-262. 
McConnell, S., Kolopack, P. \& Davis, A.M. 2001. The Western Ontario and McMaster Universities Osteoarthritis Index (WOMAC): a review of its utility and measurement properties. Arthritis Care \& Research, 45(5): 453-461.

McIntyre, M.H., Cohn, B.A. \& Ellison, P.T. 2006. Sex dimorphism in digital formulae of children. American Journal of Physical Anthropology, 129(1): 143-150.

Quinton, S.J., Smith, A.R. \& Joiner, T. 2011. The 2 to 4 digit ratio (2D:4D) and eating disorder diagnosis in women. Personality and Individual Differences, 51(4): 402-405.

Richards, G., Bellin, W. \& Davies, W. 2017. Familial digit ratio (2D:4D) associations in a general population sample from Wales. Early Human Development, 112: 14-19.

Robertson, J., Zhang, W., Liu, J.J., Muir, K.R. Maciewicz, R.A. \& Doherty, M. 2008. Radiographic assessment of the index to ring finger ratio (2D:4D) in adults. Journal of Anatomy, 212(1): 42-48.
Torres, L., Dunlop, D.D. \& Peterfy, C. 2006. The relationship between specific tissue lesions and pain severity in persons with knee osteoarthritis. Osteoarthritis and Cartilage, 14(10): 1033-1040.

White, D.K., Neogi, T., Nguyen, U.S.D.T., Niu, J. \& Zhang, Y. 2016. Trajectories of functional decline in knee osteoarthritis: the Osteoarthritis Initiative. Rheumatology, 55(5): 801-808.

Yamamotova, A., Benkova, M., Pechova, K. \& Rokyta, R. 2009. Can second to fourth digit ratio (2D:4D) predict sensitivity to pain? Activitas Nervosa Superior Rediviva, 51(3-4): 159-162.

Zhang, W., Robertson, J., Doherty, S., Liu, J.J., Maciewicz, R.A., Muir, K.R. \& Doherty, M. 2008. Index to ring finger length ratio and the risk of osteoarthritis. Arthritis \& Rheumatism, 58(1): 137-144. 
